OPEN ACCESS

Edited by:

Daniela lacoviello,

Sapienza Università di Roma, Italy

Reviewed by:

Vladislav Volman,

L-3 Communications, United States

Rodrigo Laje

Universidad Nacional de Quilmes (UNQ), Argentina

*Correspondence:

Tiago H. Falk falk@emt.inrs.ca

Received: 05 July 2017 Accepted: 15 December 2017 Published: 10 January 2018

Citation:

Clerico A, Tiwari A, Gupta $R$ Jayaraman S and Falk TH (2018) Electroencephalography Amplitude Modulation Analysis for Automated Affective Tagging of Music Video Clips. Front. Comput. Neurosci. 11:115 doi: 10.3389/fncom.2017.00115

\section{Electroencephalography Amplitude Modulation Analysis for Automated Affective Tagging of Music Video Clips}

\author{
Andrea Clerico, Abhishek Tiwari, Rishabh Gupta, Srinivasan Jayaraman and Tiago H. Falk* \\ Centre Energie, Materiaux, Telecommunications, Institut National de la Recherche Scientifique, University of Quebec, \\ Montreal, QC, Canada
}

The quantity of music content is rapidly increasing and automated affective tagging of music video clips can enable the development of intelligent retrieval, music recommendation, automatic playlist generators, and music browsing interfaces tuned to the users' current desires, preferences, or affective states. To achieve this goal, the field of affective computing has emerged, in particular the development of so-called affective brain-computer interfaces, which measure the user's affective state directly from measured brain waves using non-invasive tools, such as electroencephalography (EEG). Typically, conventional features extracted from the EEG signal have been used, such as frequency subband powers and/or inter-hemispheric power asymmetry indices. More recently, the coupling between EEG and peripheral physiological signals, such as the galvanic skin response (GSR), have also been proposed. Here, we show the importance of EEG amplitude modulations and propose several new features that measure the amplitude-amplitude cross-frequency coupling per EEG electrode, as well as linear and non-linear connections between multiple electrode pairs. When tested on a publicly available dataset of music video clips tagged with subjective affective ratings, support vector classifiers trained on the proposed features were shown to outperform those trained on conventional benchmark EEG features by as much as 6,20 , 8, and $7 \%$ for arousal, valence, dominance and liking, respectively. Moreover, fusion of the proposed features with EEG-GSR coupling features showed to be particularly useful for arousal (feature-level fusion) and liking (decision-level fusion) prediction. Together, these findings show the importance of the proposed features to characterize human affective states during music clip watching.

Keywords: emotion classification, affective computing, multimedia content, electroencephalography, physiological signals, signal processing, pattern classification

\section{INTRODUCTION}

With the rise of music and video-on-demand, as well as personalized recommendation systems, the need for accurate and reliable automated video tagging has emerged. In particular, user-centric affective tagging has stood out, corresponding to the formation of user emotional tags elicited while watching video clips (Kierkels et al., 2009; Shan et al., 2009; Koelstra and Patras, 2013). Emotions 
are usually conceived as physiological and physical responses, as part of natural communication between humans, and able to influence our intelligence, shape our thoughts and govern our interpersonal relationships (Marg, 1995; Loewenstein and Lerner, 2003; De Martino et al., 2006). Typically, machines were not required to have "emotion sensing" skills, but instead relied solely on interactivity. Recent findings from neuroscience, psychology and cognitive science, however, have modified this mentality and have pushed for such emotion sensing skills to be incorporated into machines. Such capability can allow machines to learn, in real-time, the user's preferences and emotions and adapt accordingly, thus taking the first steps toward the basic component of intelligence in human-human interaction (Preece et al., 1994).

Incorporating emotions into machines constitutes the burgeoning field of affective computing, which has as main purpose reduce the distance between the end-user and the machine by designing instruments that are able to accurately address human needs (Picard, 2000). To this end, the area of affective brain-computer interfaces (aBCIs) has recently emerged (Mühl et al., 2014). While BCIs have been mostly used to date for communication and rehabilitation applications (e.g., Li et al., 2006; Leeb et al., 2012; Sorensen and Kjaer, 2013), aBCIs (also known as passive BCIs) aim at measuring implicit information from the users, such as their moods and emotional states elicited by varying stimuli. Representative applications include neurogaming (Bos et al., 2010), neuromarketing (Lee et al., 2007), and "attention monitors" (Moore Jackson and Mappus, 2010), to name a few. As in Koelstra and Patras (2013), this paper concerns the measurement of emotions elicited on users by different music video clips, i.e., for automated multimedia tagging.

Within aBCIs, electroencephalography (EEG) has remained a popular modality due to its non-invasiveness, high temporal resolution (in the order of milliseconds), portability, and reasonable cost (Jenke et al., 2014). Typically, spectral features such as subband spectral powers have been used to measure emotional states elicited from music videos, pictures, and/or movie clips (e.g., Kierkels et al., 2009; Koelstra et al., 2012), as well as mental workload and stress (e.g., Heger et al., 2010; Kothe and Makeig, 2011). Moreover, an inter-hemispheric asymmetry in spectral power has been reported in the affective state literature (Davidson and Tomarken, 1989; Jenke et al., 2014), particularly in frontal brain regions (Coan and Allen, 2004).

Recent studies, however, have suggested that alternate EEG feature representations may exist that convey more discriminatory information over traditional spectral power and asymmetry indices (Jenke et al., 2014; Gupta and Falk, 2015). More specifically, statistical relations among temporal dynamics in different frequency bands (so-called "crossfrequency coupling") have been observed in several brain regions and are thought to reflect neural communication and information encoding to support different perceptual and cognitive processes (Cohen, 2008) and emotional states (Schutter and Knyazev, 2012). Typically, cross-frequency coupling can be measured in three ways, namely, phase-phase, phase-amplitude and amplitude-amplitude coupling. While the former two have been widely studied and shown to be related to perception and memory (e.g., theta-gamma coupling Canolty et al., 2006), the latter has received lower attention. A few studies have shown amplitude-amplitude coupling effects on personality and motivation (Schutter and Knyazev, 2012) and recently, the authors proposed an inter-hemispheric cross-frequency amplitude coupling metric that correlated with affective states (Clerico et al., 2015). Notwithstanding, existing coupling metrics typically overlook temporal dynamics and are based on interhemispheric synchrony, thus overlook synchronization of other brain regions.

Moreover, in addition to EEG correlates, affective state information has been widely obtained from physiological signals measured from the peripheral autonomic nervous system (PANS) (Nasoz et al., 2003; Lisetti and Nasoz, 2004; Wu and Parsons, 2011), particularly the galvanic skin response (GSR), a measure of the amount of sweat (conductivity) in the skin (Picard and Healey, 1997; Bersak et al., 2001). More recently, the interaction between the PANS and central nervous systems (CNS) was measured via a phase-amplitude coupling (PAC) between GSR and EEG signals and promising emotion recognition results were found for highly arousing videos (Kroupi et al., 2014). As emphasized in Canolty et al. (2012), however, different ways of computing PAC may lead to complementary information. As such, in this paper we explore different PAC computation methods to gauge the advantages of one method over another.

In this paper, we build on the work of Clerico et al. (2015) and investigate the development of alternate features based on EEG amplitude modulation analysis for automated affective tagging of music video clips. In particular, we propose a number of innovations, namely: (1) extended the inter-hemispheric crossfrequency coupling measures of EEG amplitude modulations analysis to all possible electrode pairs, thus exploring connections beyond left-right pairs, (2) explored the use of a coherence based coupling metric, as opposed to mutual information, to explore linear relationships between inter-electrode coupling, (3) explored a total amplitude modulation energy measure to capture temporal dynamics, (4) proposed a normalization scheme based on normalization of the proposed features relative to a baseline period, thus facilitating cross-subject classification (as opposed to per-subject classification in Clerico et al., 2015), and (5) explored different ways of computing PAC between EEG and GSR in order to gauge the benefits of one computation method over another. Furthermore, we show the benefits of the proposed features relative to existing spectral power-based ones, and explore their complementarity via decision- and feature-level fusion. Experimental results show the proposed features outperforming conventional ones in recognizing arousal, valence, and dominance emotional primitives, as well as a "liking" subjective parameter.

The remainder of this paper is organized as follows: Section 2 provides the methodology used, including a description of the proposed and baseline features, as well as classification and fusion strategies used. Sections 3 and 4 describe the experimental results and discusses the findings, respectively. Lastly, section 5 presents the conclusions. 


\section{MATERIALS AND METHODS}

In this section, the database, the proposed and benchmark feature sets, as well as the feature selection, classifier and classifier fusion schemes used are described.

\subsection{Affective Music Clip Audio-Visual Database}

In this paper, the publicly-available DEAP (Dataset for Emotion Analysis using EEG and Physiological signals) database was used (Koelstra et al., 2012). Thirty-two healthy subjects (genderbalanced, average age of 26.9 years) were recruited to watch 40 video music clips while their neurophysiological signals were recorded. The forty videos were carefully selected from a larger set (roughly 200 videos), corresponding to the ones eliciting the 10 highest ratings within each of the four quadrants of the valence-arousal plane (Russell, 1980). Participants were asked to rate their perceived valence, arousal, and dominance emotional primitives, as well as other subjective ratings such as liking and familiarity for each of the 40 music clips. The three emotional primitives were scored using the 9-point continuous self-assessment manikin scale (Bradley and Lang, 1994). The liking scale was introduced to determine the user's taste, and not their feelings, about the music clip; as such, 9-point scale with thumbs down/up symbols was adopted. Lastly, the familiarity rating was scored using a 5-point scale. For the purpose of this paper, the familiarity rating was not used.

Several neurophysiological signals were recorded during music clip watching, namely 32-channel EEG (Biosemi Active II, with 10-20 international electrode placement), skin temperature, GSR, respiration, and blood volume pulse. The raw signals were recorded at a $512 \mathrm{~Hz}$ sample rate and down sampled offline to $128 \mathrm{~Hz}$. The EEG signals were further bandpass filtered from 4 to $45 \mathrm{~Hz}$, pre-processed using principal component analysis to remove ocular artifacts, averaged to a common reference and made publicly available. The interested reader is referred to Koelstra et al. (2012) for more details about the database.

\subsection{Feature Extraction}

\subsubsection{Spectral Features}

Spectrum subband power features are the most traditional measures used in biomedical signal processing (Sörnmo and Laguna, 2005). Within the affective state recognition literature, spectral power in the theta $(4-8 \mathrm{~Hz})$, alpha $(8-12 \mathrm{~Hz})$, beta $(12-30$ $\mathrm{Hz}$ ), and gamma $(30-45 \mathrm{~Hz})$ subbands are typically used (Jenke et al., 2014) across different brain regions (Schutter et al., 2001; Balconi and Lucchiari, 2008). In particular, alpha and gamma band inter-hemispheric asymmetry indices have been shown to be correlated with emotional ratings, particularly in frontal brain regions (Müller et al., 1999; Mantini et al., 2007; Arndt et al., 2013). Given their widespread usage and the fact that they were also used in Koelstra et al. (2012) for affect recognition from the DEAP database, spectral features ("SF") are used here as a benchmark to gauge the benefits of the proposed features. A total of 128 spectral power features (32 electrodes $\times 4$ subbands) and 56 asymmetry indices (14 inter-hemispheric pairs $\times 4$ subbands) were computed from the following electrode pairs: Fp1-Fp2,
AF3-AF4, F7-F8, F3-F4, FC5-FC6, FC1-FC2, T7-T8, C3-C4, CP5-CP6, CP1-CP2, P7-P8, P3-P4, PO3-PO4, and O1-O2 (see Figure 1 for electrode labels and locations). Overall, a total of 184 "SF" features are used as benchmark.

\subsubsection{Amplitude Modulation Features}

Cross-frequency amplitude-amplitude coupling in the EEG has been explored in the past as a measure of anxiety and motivation (e.g., Schutter and Knyazev, 2012), but has been under-explored within the affective state recognition community. Recently, betatheta amplitude-amplitude coupling differences were observed between healthy elderly controls and age-matched Alzheimer's disease patients; such findings were linked to lack of interest and motivation within the patient population (Falk et al., 2012). To explore the benefits of cross-frequency amplitude-amplitude modulations for affective state recognition research, the authors recently showed that non-linear coupling patterns within interhemispheric electrode pairs was a reliable indicator of several affective dimensions, but particularly for the valence emotional primitive (Clerico et al., 2015). In this paper, we extend this work by extracting a number of other amplitude modulation features ("AMF") and show their advantages for affective state recognition.

More specifically, three new amplitude-amplitude coupling feature sets are extracted, namely the amplitude modulation energy (AME), amplitude modulation interaction (AMI), and the amplitude modulation coherence (AMC), as depicted by Figure 1. In order to compute these three feature sets, first the full-band EEG signal $s_{k}$ for channel "k" (see left side of the figure) is decomposed into the four typical subbands (theta, alpha, beta and gamma) using zero-phase digital bandpass filters. Here, the time-domain index " $n$ " is omitted for brevity, but without loss of generality. For the sake of notation, the decomposed timedomain signal is referred to as $s_{k}(i), i=1, \ldots, 4$. The temporal envelope is then extracted from each of the four subband time series using the Hilbert transform (Le Van Quyen et al., 2001). Figure 2 illustrates the extracted EEG subband time series in gray and their respective Hilbert amplitude envelopes in black. Here, the temporal envelopes $e_{i}(n)$ of each subband time series were computed as the magnitude of the complex analytic signal $\zeta(n)=s_{k}(i)^{2}+j \mathcal{H}\left\{s_{k}(i)\right\}$, i.e.,

$$
e_{i}(i)=\sqrt{s_{k}(i)^{2}+\mathcal{H}\left\{s_{k}(i)\right\}^{2}},
$$

where, $\mathcal{H}\{\cdot\}$ corresponds to the Hilbert transform.

In order to measure cross-frequency amplitude-amplitude coupling, a second decomposition of the EEG amplitude envelopes is performed utilizing the same four subbands. To distinguish between modulation and frequency subbands, the former are referred to as $\mathrm{m}-\theta(4-8 \mathrm{~Hz}), \mathrm{m}-\alpha(8-12 \mathrm{~Hz}), \mathrm{m}-\beta$ $(12-30 \mathrm{~Hz})$ and $\mathrm{m}-\gamma(30-45 \mathrm{~Hz})$. For notation, the amplitudeamplitude coupling pattern is termed $s_{k}(i, j), i, j=1, \ldots, 4$, where "i" indexes spectral subbands and " $j$ " the modulation spectral subbands. By using the Hilbert transform to extract the amplitude envelope, the types of cross-frequency interactions are limited by Bedrosian's theorem, which states that the envelope signals can only contain frequencies (i.e., modulated frequencies) up to the 


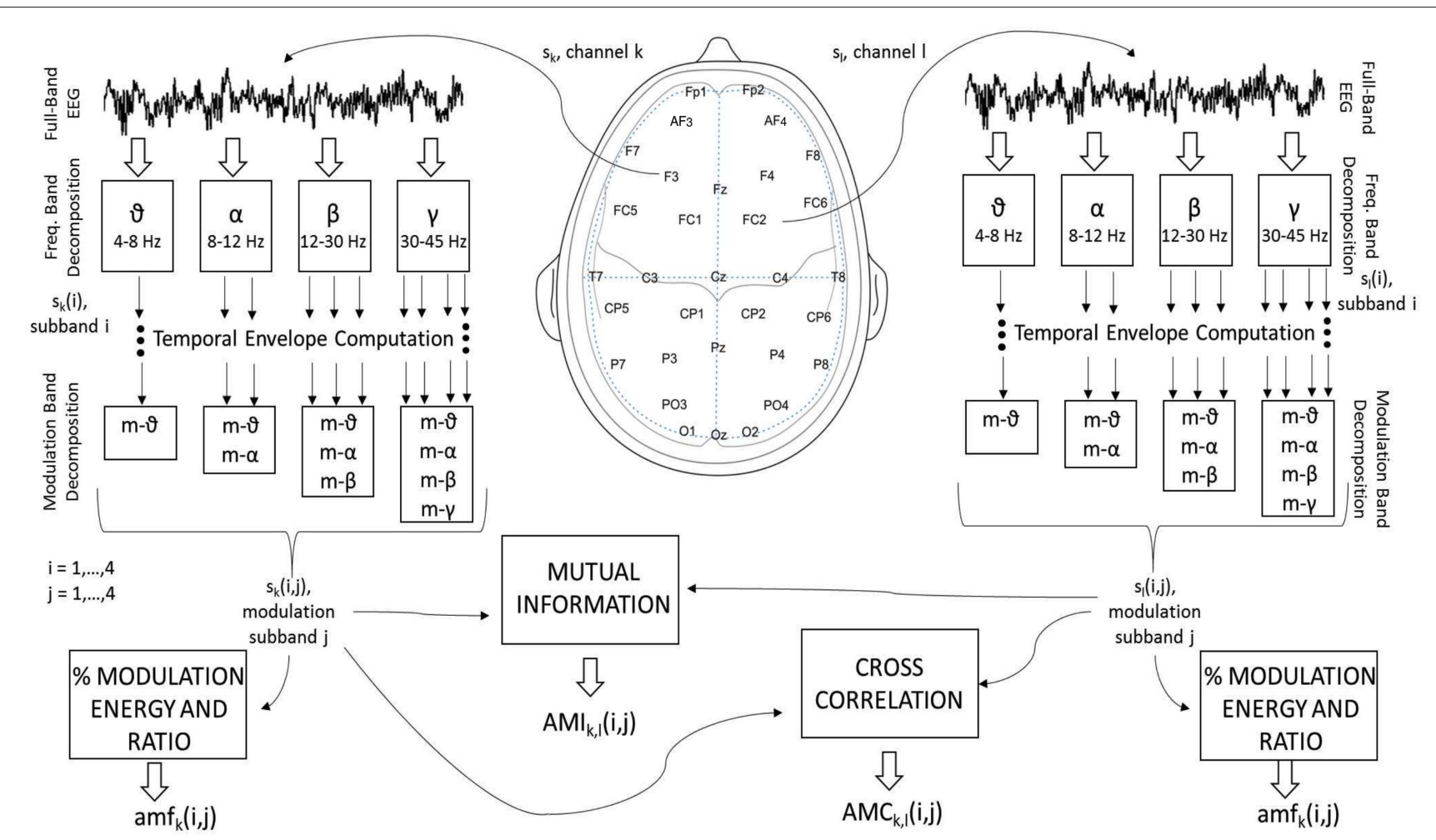

FIGURE 1 | Signal processing steps used to compute the EEG amplitude modulation feature sets.

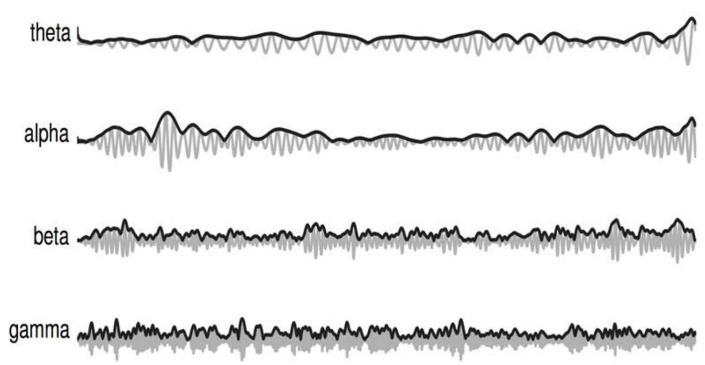

FIGURE 2 | Amplitude envelope extraction from each EEG subband time series signal (gray) and their respective Hilbert amplitude envelopes (black).

maximum frequency of its original signal (Boashash, 1991; Smith et al., 2002). As such, only the ten cross-frequency patterns shown in Figure 1 are possible (per electrode), namely: $\theta \_\mathrm{m}-\theta, \alpha \_\mathrm{m}-\theta$, $\alpha \_\mathrm{m}-\alpha, \beta \_\mathrm{m}-\theta, \beta \_\mathrm{m}-\alpha, \beta \_\mathrm{m}-\beta, \gamma \_\mathrm{m}-\theta, \gamma \_\mathrm{m}-\alpha, \gamma \_\mathrm{m}-\beta$, and $\gamma \_\mathrm{m}-\gamma$. From these patterns, the three feature sets are computed, as detailed below:

\subsubsection{Amplitude modulation energy ( $A M E)$}

From the ten possible $s_{k}(i, j)$ patterns per electrode, two energy measures are computed. The first measures the ratio of energy in a given frequency-modulation-frequency pair $\left(\xi_{k}(i, j)\right)$ over the total energy across all possible subbands pair (i.e., $\left.\sum_{i=1}^{4} \sum_{j=1}^{4} \xi_{k}(i, j)\right)$, thus resulting in 320 features (32 electrodes $\times 10$ cross-frequency coupling patterns; see possible combinations in Figure 1). The second measures the logarithm of the ratio of modulation energy during the 60-s music clip to the modulation energy during a 3 -s baseline resting period, i.e., $10 \log \left(\xi_{k}(i, j)^{\text {video }} / \xi_{k}(i, j)^{\text {baseline }}\right)$, thus resulting in an additional 320 features, for a total of $640 \operatorname{AME}_{k}(i, j)$ features, $k=$ $1, \ldots, 32 ; i, j=1, \ldots, 4$.

\subsubsection{Amplitude modulation interaction (AMI)}

In order to incorporate inter-electrode amplitude modulation (non-linear) synchrony, the amplitude modulation interaction (AMI) features from Clerico et al. (2015) are also computed. Unlike the work described in Clerico et al. (2015), where interactions were only computed per symmetric interhemispheric pairs, here we measure interactions across all possible 496 electrode pair combinations (i.e., 2-by-2 combinations over all possible 32 channels) for each of the ten cross-frequency coupling patterns, thus resulting in 4960 features. The normalized mutual information (MI) is used to measure the interaction:

$$
A M I_{k, l}=\frac{H\left(s_{k}\right)+H\left(s_{l}\right)-H\left(s_{k}, s_{l}\right)}{\sqrt{H\left(s_{k}\right) H\left(s_{l}\right)}},
$$

where the $H(\cdot)$ operator represents marginal entropy and $H(\cdot, \times)$ the joint entropy, and $s_{k}$ corresponds to $s_{k}(i, j)$ with the 
frequency and modulation frequency indices omitted for brevity. Entropy was calculated using the histogram method with 50 discrete bins for each variable. Mutual information has been used widely in affective recognition research (e.g., Cohen et al., 2003; Khushaba et al., 2012; Hamm et al., 2014). Additionally a second measurement of logarithmic ratio between the 60-s clip and the 3-s baseline has been obtained, thus totalling 9920 AMI features.

\subsubsection{Amplitude modulation coherence (AMC)}

While the AMI features capture non-linear interactions between inter-electrode amplitude-amplitude coupling patterns, the Pearson correlation coefficient between the patterns can also be used to quantify the coherence, or linear interactions between the patterns. Spectral coherence measures have been widely used in EEG research and were recently shown to also be useful for affective state research (e.g., Kar et al., 2014; Xielifuguli et al., 2014). Hence, we explore the concept of amplitude modulation coherence, or AMC as a new feature for affective state recognition. The AMC features are computed as:

$$
A M C_{k, l}=\frac{\sum_{n=1}^{N}\left(s_{k}(n)-\overline{s_{k}}\right)\left(s_{l}(n)-\bar{s}_{l}\right)}{\sqrt{\sum_{n=1}^{N}\left(s_{k}(n)-\overline{s_{k}}\right)^{2} \sum_{n=1}^{N}\left(s_{l}(n)-\overline{s_{l}}\right)^{2}}}
$$

where $s_{k}(n)$ indicates the $\mathrm{n}$-th sample of the $s_{k}(i, j)$ time-series (again, the frequency and modulation frequency indices were omitted for brevity), and $\overline{s_{k}}$ is the average over all samples of such time series. As previously, a total of 9920 AMC features are computed, including the logarithmic ratio with the 3 -s baseline.

\subsubsection{PANS-CNS Phase-Amplitude Coupling (PAC)}

Electrophysiological signals reflect dynamical systems that interact with each other at different frequencies. PhaseAmplitude coupling represents one type of interaction and typically refers to modulation of the amplitude of highfrequency oscillators by the phase of low-frequency ones (Samiee et al.). Typically, such phase-amplitude coupling measures are computed from EEG signals alone (Schutter and Knyazev, 2012), but the concept of electrodermal activity phase coupled to EEG amplitude was recently introduced as a correlate of emotion, particularly for high arousing, very pleasant and very unpleasant stimuli (Kroupi et al., 2013, 2014). Here, we test three different GSR-phase and EEG-amplitude coupling measures. For the sake of notation, assume $u(n)$ is the rapid transient response called skin conductance response (SCR) with a narrowband of $0.5-1 \mathrm{~Hz}$ (Kroupi et al., 2014), of the time-domain GSR signal. Using the Hilbert transform (Gabor, 1946), we can extract the signal's instantaneous phase $\phi_{u}(n)$ as in Kroupi et al. (2014):

$$
\phi_{u}(n)=\arctan \left(\frac{\mathcal{H}\{u(n)\}}{u(n)}\right) .
$$

For the amplitude envelope of the EEG signal $\left(A\left(s_{k}(n)\right)\right)$, a shapepreserving piecewise cubic interpolation method of neighboring values is used, as in Kroupi et al. (2014). Given the GSR signal and phase, as well as the EEG amplitude envelope signals, the following coupling measures were computed.

\subsubsection{Envelope-to-signal coupling (ESC)}

The simplest coupling feature can be calculated via the Pearson correlation coefficient between the EEG amplitude envelope signal $A\left(s_{k}(n)\right)$ and the raw GSR signal $u(n)$. The ESC feature can be computed using equation (3) with $A\left(s_{k}(n)\right)$ and $u(n)$ in lieu of $s_{k}(i, j)$ and $s_{l}(i, j)$, respectively (Arnulfo et al., 2015). ESC has been shown to be particularly useful with noisy data (Onslow et al., 2011). A total of 32 ESC features were computed.

\subsubsection{Cross-frequency coherence (CFC)}

Cross-frequency coherence evaluates the magnitude square coherence between the filtered $(0-1 \mathrm{~Hz})$ GSR signal $u(n)$ and the filtered $(4-45 \mathrm{~Hz})$ envelope of the EEG signal $A\left(s_{k}(n)\right)$, as in Onslow et al. (2011). The CFC feature is computed as:

$$
\operatorname{CFC}_{k}(f)=\frac{\left|P_{A u}(f)\right|^{2}}{P_{A A}(f) P_{u u}(f)}
$$

where $\left|P_{A u}(f)\right|^{2}$ is the cross power spectral density of the EEG amplitude $A\left(s_{k}(n)\right)$ and GSR signal $u(n)$ at frequency $f$, and $P_{A A}(f)$ and $P_{u u}(f)$ are the spectral power densities of the two signals, respectively. The CFC feature ranges from 0 (no spectral coherence) to 1 (perfect spectral coherence) and has been used previously to quantify linear EEG synchrony in different frequency bands and its relationship with emotions (Daly et al., 2014). A total of 1344 CFC features were computed.

\subsubsection{Modulation index (ModI)}

PANS-CNS coupling measure tested is the so-called modulation index (ModI), which was recently shown to accurately characterize coupling intensity (Tort et al., 2010), particularly for emotion recognition (Kroupi et al., 2014). For calculation of the ModI feature, a composite times series is constructed as $\left[\phi_{u}(n), A\left(s_{k}(n)\right)\right]$. The phases are then binned and the mean of $A\left(s_{k}(n)\right)$ over each phase bin is calculated and denoted by $\left\langle A_{s}\right\rangle \phi_{u}(m)$, where $\mathrm{m}$ indexes phase bin; 18 bins were used in this experiment. Further, the mean amplitude distribution $P(m)$ is normalized by the sum over all bins, i.e.,:

$$
P(m)=\frac{\left\langle A_{s}\right\rangle \phi_{u}(m)}{\sum_{m=1}^{18}\left\langle A_{s}\right\rangle \phi_{u}(m)} .
$$

The normalized amplitude "distribution" $P(m)$ has similar properties as a probability density function. In fact, in the scenario in which no phase-amplitude coupling exists, $P(n)$ assumes a uniform distribution. Having this said, the ModI feature measures the deviation of $P(m)$ from a uniform distribution. This is achieved by means of a Kullback-Liebler (KL) divergence measure (Kullback and Leibler, 1951) between $P(m)$ and a uniform distribution $Q(m)$, given by:

$$
D_{K L}(P, Q)=\sum_{m=1}^{18} U(m) \log \left[\frac{P(m)}{Q(m)}\right]
$$

The KL divergence $D_{K L}(P, Q)$ is always greater than zero, and equal to zero only when the two distributions are the same. 
Finally, the ModI feature is defined as the ratio between the KL divergence and the log of the number of phase bins, i.e.,:

$$
M o d I=\frac{D_{K L}(P, Q)}{\log (M)} .
$$

where $M=18$ is used in our experiments. A total of 32 ModI features were computed.

\subsection{Feature Selection and Affective State Recognition}

In this section, a description of the feature selection, classifiers, and classifier fusion strategies are discussed.

\subsubsection{Feature Selection}

As mentioned above, a large number of proposed and benchmark features were extracted. More specifically, a total of 184 SF, 20480 AMF, and 1408 PAC features were extracted. For classification purposes, these numbers are large and may lead to classifier overfitting. In such instances, feature ranking and/or feature selection algorithms are typically used. Recently, several feature selection algorithms were compared on an emotion recognition task (Jenke et al., 2014). The minimum redundancy maximum relevance (mRMR) algorithm (Peng et al., 2005) showed improved performance when paired with a support vector machine classifier (Wang et al., 2011). The mRMR is a mutual information based algorithm that optimizes two criteria simultaneously: the maximum-relevance criterion (i.e., maximizes the average mutual information between each feature and the target vector) and the minimum-redundancy criterion (i.e., minimizes the average mutual information between two chosen features). The algorithm finds near-optimal features using forward selection with the chosen features maximizing the combined max-min criteria.

Moreover, in an allied domain, multi-stage feature selection comprised of analysis of variance (ANOVA) between the features and target labels as a pre-screening, followed by mRMR, was shown to lead to improved results for SVM-based classifiers (Dastgheib et al., 2016). This multi-stage feature selection procedure is explored herein and during pre-screening, only features that attained $p$-values smaller than 0.1 were kept. Here, two tests are explored. With one, all top selected features for each feature class are used for classifier training. Given the different number of available features for each feature class, the input dimensionality of the attained classifiers will differ. For a more fair comparison, the second assumes that classifiers are trained on the same number of features for each feature class. To this end, the number of features used corresponds to the number of benchmark SF features that pass the ANOVA test.

In the available dataset, neurophysiological signals were recorded from 32 subjects while each watched a total of 40 music clips. Here, $25 \%$ of the available data (i.e., data from 10 music clips per subject, roughly half from the high and half from the low classes) was set aside for feature ranking. The remaining $75 \%$ was used for classifier training and testing in a leave-one-sample-out
(LOSO) cross-validation scheme, as described next. This holdout scheme assures a more stringent setup, as feature selection and model training are not performed on the same data subset, which could lead to overly optimistic results. From the feature selection set, it was found that $35,23,19$, and 21 SF features passed the ANOVA test for arousal, valence, dominance, and liking dimensions, respectively.

\subsubsection{Classification}

During pilot phase, support vector machine (SVM), relevance vector machine (RVM) and random forest classifiers were explored. Overall, SVMs resulted in improved performance. Indeed, they have been widely used in bioengineering and in affective state recognition (e.g., Wang et al., 2011). Given their widespread use, a description of the support vector machine approach is not included here and the interested reader is referred to Schölkopf and Smola (2002) and references therein for more details. Here, SVM classifiers are trained on four different binary classification problems, i.e., detecting low/high valence, low/high arousal, low/high dominance and low/high liking.

With the DEAP database, subjective ratings followed a 9point scale. Typically, values greater or equal to 5 are assumed to correspond to high activation levels or low, otherwise. However, it is not guaranteed that all users objectively utilize the same scale for grading. In fact, by using a threshold of 5, a 60/40 ratio of high/low levels was obtained across all participants. In order to take into account individual biases during rating, here we utilize an individualized threshold corresponding to the value in which an almost balanced high/low ratio was achieved per participant. Figure 3 depicts the threshold found for each participant for arousal and valence. As can be seen, on average a threshold of 5 was most often selected, though in a few cases, much higher or much lower values were found, thus exemplifying the need for such an individualized approach.

As mentioned previously, $75 \%$ of the available dataset was used for classifier training/testing using a leave-one-sample-out (LOSO) cross-validation scheme. For our experiments, a radial basis function (RBF) kernel was used and implemented with the Scikit-learn library in Python (Pedregosa et al., 2011). Since we are interested in gauging the benefits of the proposed features, and not of the classification schemes, we use the default SVM parameters throughout our experiments (i.e., $\lambda=1$ and $\gamma_{R B F}=$ $0.01)$. As such, it is expected that improved performance should be achieved once classifier optimization is performed, as in Gupta et al. (2016). Such analysis, however, is left for future study.

\subsubsection{Fusion}

In an attempt to improve classification performance, two fusion strategies are explored, namely, feature fusion and decision-level fusion. In feature fusion, we explore the combination of the three feature sets (SF, PAC, and AMF) and utilize the top selected features. With classifier decision-level fusion, on the other hand, the decisions of the three SVM classifiers trained on the top SF, $\mathrm{PAC}$, and AMF sets were fused using a simple majority voting scheme with equal weights. 


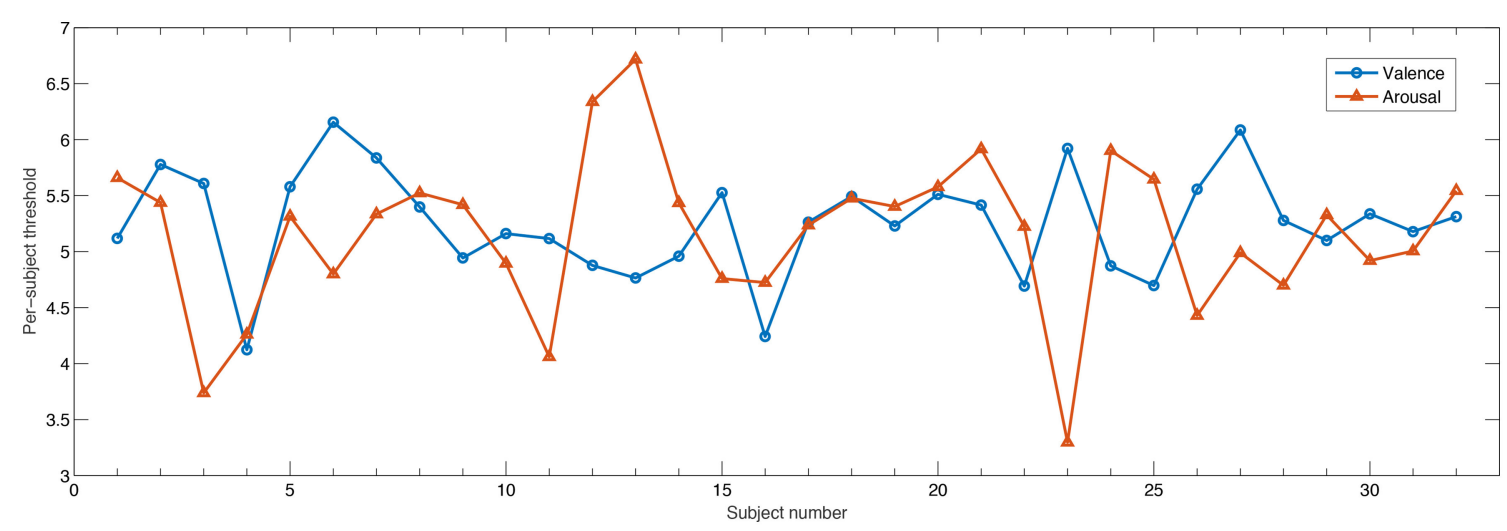

FIGURE 3 | Individualized threshold such that approximately 50/50 ratio was achieved for high/low class for valence and arousal dimensions.

\subsection{Figure of Merit}

Balanced accuracy (BACC) is used as a figure of merit and corresponds to the arithmetic mean of the classifier sensitivity and specificity, namely:

$$
B A C C=\frac{S E N S+S P E C}{2},
$$

where

$$
S E N S=\frac{T P}{P} ; S P E C=\frac{T N}{N},
$$

and $P=T P+F N$ and $N=F P+T N$, TP and FP correspond to true and false positives, respectively and $\mathrm{TN}$ and $\mathrm{FN}$ to true and false negatives, respectively. Balanced accuracy takes into account any remaining class unbalances and provides more accurate results than the conventional accuracy metric. To test the significance of the attained performances, an independent one-sample $t$-test against a random voting classifier was used $(p<0.05)$, as suggested in Koelstra et al. (2012).

\section{RESULTS}

Tables 1-4 show the top-selected features for the arousal, valence, dominance, and liking dimensions, respectively, following multistage feature selection and using the same number of features across sets. Feature names listed in the tables should be self explanatory. The "ratio" features correspond to the log-ratio ones between the video and baseline periods (see section 2.2.2). In the SF category, the "AI" features correspond to the asymmetry index between the indicated channels.

Table 5, in turn, reports the balanced accuracy results achieved with the individual features sets and the same dimensionality, as well as with the feature- and decisionlevel fusion strategies. All obtained results were significantly higher $(p<0.05)$ than those achieved with a random voting classifier (Koelstra et al., 2012). The column labeled "\%" indicates the relative improvement in balanced accuracy, in percentage, relative to the SF baseline set. As can be seen, all proposed AMF features outperform the benchmark, by as much as 4.4 ,
5.6, 5.6, and $1.9 \%$ for valence, arousal, dominance, and liking, respectively. The PAC features also show advantages over the benchmark, particularly for the valence dimension, in which a $9.7 \%$ gain was observed. Feature fusion, in turn, showed to be useful mostly for arousal prediction, whereas decision-level fusion was useful for the liking dimension.

Moreover, for classifiers of varying dimensionality, maximum balanced accuracy values of 0.625 (AMI), 0.652 (AME), 0.659 (AMC) could be achieved for valence, dominance, liking, respectively, thus representing gains over the benchmark set of 8.1, 20.3, and 6.5\%. For PAC features, gains could be seen only for the dominance dimension where a balanced accuracy of 0.592 could be seen, representing a gain over SF of $9.2 \%$.

\section{DISCUSSION}

\subsection{Feature Ranking}

From Tables 1-4, it can be seen that with the exception of arousal, the number of SF features that passed the pre-screening test was roughly 20. For valence, roughly half those features corresponded to asymmetry index features, and across most emotional primitives, $\alpha, \beta$ and $\theta$ frequency bands showed to be the most relevant. These findings corroborate those widely reported in the literature (e.g., Davidson et al., 1979; Hagemann et al., 1999; Coan and Allen, 2004; Davidson, 2004).

Previous work on PAC, in turn, showed the coupling between EEG and GSR (computed via the ModI feature) to be relevant in emotion classification, particularly for arousal and valence (Kroupi et al., 2014). Interestingly, the CFC method of computing PANS-CNS phase-amplitude coupling was most often selected; for arousal $97 \%$ of the top features corresponded to CFC-type features. ModI features, in fact, were never selected as being a top candidate. PAC features showed to be particularly useful for valence estimation where $80 \%$ of the top features emanated from central brain regions $(\mathrm{C} 3, \mathrm{CP} 1, \mathrm{FC} 1)$ and the attained balanced accuracy outperformed all other tested features. Such findings suggest that alternate PAC representations should be explored, especially within the scope of valence estimation. 
TABLE 1 | Selected top-35 features for the arousal dimension.

\begin{tabular}{|c|c|c|c|c|c|}
\hline Ranking & \multicolumn{5}{|c|}{ Arousal } \\
\hline 2 & $\beta \_\mathrm{m}-\alpha \_\mathrm{FC} 5$ _CP5 & $\theta \_\mathrm{m}-\theta \_\mathrm{Fp} 1 \_\mathrm{Pz}$ & $\beta \_\mathrm{m}-\alpha \_\mathrm{F} 7$ & cfc_CP5_7_Hz & Al_ $\theta$ FC1_FC2 \\
\hline 3 & $\gamma_{-} \mathrm{m}-\gamma_{-} \mathrm{FC} 5 \_\mathrm{Cz}$ & $\gamma_{-} \mathrm{m}-\gamma_{-}$FC5_FC1 & ratio_$\gamma \_\mathrm{m}-\beta \_\mathrm{Pz}$ & cfc_O1_19_Hz & $\gamma_{-} \mathrm{Fp} 1$ \\
\hline 4 & $\gamma_{-} \mathrm{m}-\gamma_{-} \mathrm{FC5} \_\mathrm{AF4}$ & $\gamma \_\mathrm{m}-\theta_{-} \mathrm{CP5}$ _F8 & $\theta \_\mathrm{m}-\theta \_\mathrm{O} 1$ & cfc_FC5_15_Hz & $\theta \_\mathrm{O} 2$ \\
\hline 6 & $\beta \_\mathrm{m}-\alpha \_\mathrm{CP} 5 \_\mathrm{Pz}$ & $\alpha \_\mathrm{m}-\alpha \_\mathrm{P} 7 \_\mathrm{C} 4$ & $\beta \_\mathrm{m}-\beta \_\mathrm{O} 2$ & cfc_O1_20_Hz & $\alpha \_F 7$ \\
\hline 7 & $\gamma_{-} \mathrm{m}-\gamma_{-}$FC5_PO4 & $\alpha \_\mathrm{m}-\theta \_F 7 \_T 7$ & ratio_ $\alpha \_m-\theta \_O 2$ & cfc_O1_27_Hz & $\theta_{-} \mathrm{CP} 6$ \\
\hline 8 & $\gamma_{-} \mathrm{m}-\alpha_{-} \mathrm{PO}$ _F8 & $\gamma \_\mathrm{m}-\gamma_{-}$P7_F8 & $\gamma \_\mathrm{m}-\beta \_\mathrm{F} 7$ & cfc_O1_28_Hz & $\alpha \_\mathrm{Pz}$ \\
\hline 9 & $\gamma_{-} \mathrm{m}-\gamma_{-} \mathrm{FC5} \_\mathrm{C} 4$ & $\beta \_\mathrm{m}-\theta \_\mathrm{C} 4 \_\mathrm{P} 4$ & ratio_$\alpha \_m-\alpha \_$_8 & cfc_FC5_16_Hz & Al_$\beta \_A F 3 \_A F 4$ \\
\hline 10 & $\gamma_{-} \mathrm{m}-\beta_{-}$FC5_PO4 & $\theta \_\mathrm{m}-\theta_{-} \mathrm{FC} 6 \_\mathrm{Cz}$ & ratio_ $\beta \_\mathrm{m}-\beta_{-} \mathrm{FC} 2$ & cfc_FC1_39_Hz & $\beta_{-}$FC5 \\
\hline 11 & $\beta \_\mathrm{m}-\theta \_\mathrm{FC} 2 \_\mathrm{P} 8$ & $\alpha \_\mathrm{m}-\theta_{-}$T8_CP6 & $\theta \_\mathrm{m}-\theta_{-} \mathrm{FC} 5$ & cfc_FC1_43_Hz & $\theta \_$AF4 \\
\hline 15 & $\beta \_\mathrm{m}-\beta \_$AF3_CP5 & $\alpha \_\mathrm{m}-\theta \_\mathrm{P} 7 \_\mathrm{C} 4$ & ratio_ $\alpha \_m-\alpha \_O 2$ & cfC_P8_5_Hz & Al_ $\beta \_F C 5 \_F C 6$ \\
\hline 16 & $\beta \_\mathrm{m}-\beta \_\mathrm{FC} 5 \_\mathrm{CP} 5$ & $\beta \_\mathrm{m}-\alpha \_\mathrm{C} 4 \_\mathrm{P} 4$ & ratio_$\alpha \_m-\theta \_F z$ & cfC_FC1_37_Hz & $\beta_{-} \mathrm{Fp} 2$ \\
\hline 17 & $\alpha \_\mathrm{m}-\alpha \_\mathrm{FC} 1 \_\mathrm{T} 8$ & $\theta$ _m- $\theta$ _C3_O1 & ratio_ $\alpha \_m-\alpha \_C z$ & cfc_01_29_Hz & $\theta_{-}$FC6 \\
\hline 18 & $\alpha \_\mathrm{m}-\alpha_{-} \mathrm{O} z \_\mathrm{CP} 2$ & $\theta \_\mathrm{m}-\theta \_$P3_P8 & $\gamma \_\mathrm{m}-\alpha_{-} \mathrm{F} 7$ & cfc_01_23_Hz & $\theta_{-}$T8 \\
\hline 19 & $\gamma_{-} \mathrm{m}-\gamma_{-}$FC5_FC6 & $\alpha \_\mathrm{m}-\theta$-Fp1_Cz & $\alpha \_\mathrm{m}-\theta \_\mathrm{O} 2$ & cfc_01_22_Hz & $\alpha \_F z$ \\
\hline 20 & $\beta \_\mathrm{m}-\beta \_\mathrm{PO} 3 \_\mathrm{P} 8$ & $\gamma \_\mathrm{m}-\alpha_{-} T 7$ FC2 & ratio_$\beta \beta \_\mathrm{m}-\theta \_\mathrm{P} 3$ & cfc_FC1_8_Hz & $\alpha \_\mathrm{PO} 3$ \\
\hline 21 & $\gamma \_\mathrm{m}-\beta \_\mathrm{AF} 4 \_\mathrm{PO} 4$ & $\gamma \_\mathrm{m}-\beta \_\mathrm{FC} 5$ FCC1 & $\alpha \_\mathrm{m}-\theta_{-} \mathrm{T} 8$ & cfC_FC5_18_Hz & $\gamma_{-} \mathrm{F} 4$ \\
\hline 22 & $\gamma \_\mathrm{m}-\beta_{-} \mathrm{FC5} \_\mathrm{Fz}$ & $\beta \_\mathrm{m}-\theta_{-} \mathrm{T} 7 \_\mathrm{T} 8$ & ratio_$\gamma_{-} \_\mathrm{m}-\gamma_{-} \mathrm{Oz}$ & cfc_FC1_35_Hz & Al_ $\theta$ _O1_O2 \\
\hline 23 & $\beta \_\mathrm{m}-\alpha \_\mathrm{AF} 3 \_\mathrm{Pz}$ & $\gamma \_\mathrm{m}-\alpha_{-} \mathrm{FC} 5$ FC1 & $\theta \_\mathrm{m}-\theta \_\mathrm{P} 7$ & cfc_Fz_19_Hz & $\theta \_\mathrm{P} 8$ \\
\hline 24 & $\gamma \_\mathrm{m}-\gamma_{-} \mathrm{AF} 4 \_\mathrm{PO} 4$ & $\beta \_\mathrm{m}-\theta_{-} \mathrm{Cz} \_\mathrm{PO} 4$ & ratio_$\gamma \_\mathrm{m}-\theta_{-} \mathrm{CP} 1$ & esc_C4 & Al_$\beta$ _Fp1_Fp2 \\
\hline 32 & $\alpha \_\mathrm{m}-\theta$-F3_Oz & $\gamma \_\mathrm{m}-\beta \_\mathrm{CP5} 5$ F8 & $\alpha \_\mathrm{m}-\theta \_\mathrm{Cz}$ & cfC_FC5_19_Hz & $\alpha \_\mathrm{P} 7$ \\
\hline 33 & $\gamma \_\mathrm{m}-\beta \_\mathrm{FC5}$-FC6 & $\gamma \_\mathrm{m}-\beta \_\mathrm{P} 7 \_\mathrm{F} 8$ & ratio_$\theta-\mathrm{m}-\theta \_\mathrm{O} 2$ & cfc_O1_21_Hz & $\mathrm{Al} \_\beta \_\mathrm{F} 7 \_\mathrm{F} 8$ \\
\hline 34 & $\gamma_{-} \mathrm{m}-\gamma_{-} \mathrm{F} 3 \_\mathrm{Fp} 2$ & $\alpha \_\mathrm{m}-\alpha \_F 7 \_T 7$ & $\beta \_\mathrm{m}-\beta \_\mathrm{F} 7$ & cfc_FC5_17_Hz & $\beta \_$AF3 \\
\hline 35 & $\gamma \_\mathrm{m}-\gamma_{-} \mathrm{FC} 1 \_\mathrm{AF} 4$ & $\alpha \_\mathrm{m}-\alpha \_F p 1 \_\mathrm{Cz}$ & ratio_ $\alpha \_m-\theta \_A F 4$ & cfc_O1_24_Hz & $\theta \_\mathrm{CP} 2$ \\
\hline
\end{tabular}

Feature names listed should be self explanatory. The "ratio" features correspond to the log-ratio ones between the video and baseline periods; "Al" corresponds to the asymmetry index between the indicated channels.

Regarding the proposed AMF features, for arousal estimation, $\gamma$ and $\beta$ bands showed to be particularly useful, corresponding to roughly $86 \%$ of the top AMI features and $50 \%$ of the AMC and AME features. These findings are inline with results from Jenke et al. (2014). For valence, $\alpha$ interactions showed to be particularly useful, appearing in roughly $70 \%$ of the top AMI features. In particular $\alpha \_\mathrm{m}-\theta$ interactions stood out, thus corroborating previous findings (Kensinger, 2004) which related these bands to states of internalized attention and positive emotional experience (Aftanas and Golocheikine, 2001). Such alpha/theta cross-frequency synchronization has also been previously related to memory usage (Chik, 2013). To corroborate this hypothesis, the correlation between the proposed features derived from the $\alpha \_\mathrm{m}-\theta$ patterns and the subjective "familiarity" ratings reported by the participants was computed. The majority of the features showed to be significantly correlated $(\geq 0.35, p<$ 0.05 ) with the familiarity rating, thus suggesting memory may have indeed played an effect on the elicited affective states.

Moreover, it was previously demonstrated that the power in the $\gamma$ and $\beta$ bands were also able to discriminate between liking and disliking judgements (Hadjidimitriou and Hadjileontiadis, 2012). By analyzing their amplitude modulation cross-frequency coupling via the proposed features, improved results were observed, thus showing the importance of EEG amplitude 
TABLE 2 | Selected top-23 features for the valence dimension.

\begin{tabular}{|c|c|c|c|c|c|}
\hline Ranking & \multicolumn{5}{|c|}{ Valence } \\
\hline 2 & $\alpha \_\mathrm{m}-\alpha \_01 \_\mathrm{Oz}$ & $\beta \_\mathrm{m}-\beta \_\mathrm{AF} 3 \_\mathrm{F} 4$ & ratio_$\gamma \_\mathrm{m}-\alpha \_\mathrm{PO} 3$ & cfc_C3_26_Hz & $\alpha \_\mathrm{P} 7$ \\
\hline 3 & $\alpha \_\mathrm{m}-\theta \_\mathrm{F} 7 \_\mathrm{Pz}$ & $\gamma_{-} \mathrm{m}-\gamma_{-} \mathrm{CP} 1 \_\mathrm{P} 7$ & ratio_$\beta \_\mathrm{m}-\beta \_\mathrm{Fp} 1$ & cfc_CP1_25_Hz & $\gamma_{-} F z$ \\
\hline 4 & $\alpha \_\mathrm{m}-\alpha_{-} \mathrm{F} 3 \_\mathrm{O} 1$ & $\gamma \_\mathrm{m}-\theta$ _AF3_Oz & ratio_$\alpha \_m-\theta \_O z$ & cfc_CP1_28_Hz & $\alpha \_\mathrm{P} 3$ \\
\hline 6 & $\alpha \_\mathrm{m}-\alpha \_01 \_\mathrm{O} 2$ & $\gamma \_\mathrm{m}-\beta \_\mathrm{F} 3 \_\mathrm{Oz}$ & $\beta \_\mathrm{m}-\theta \_\mathrm{Pz}$ & cfc_C3_25_Hz & $\mathrm{Al} \_\gamma \_01 \_\mathrm{O} 2$ \\
\hline 7 & $\alpha \_\mathrm{m}-\alpha \_T 7 \_\mathrm{O} 1$ & $\gamma \_\mathrm{m}-\gamma \_\mathrm{AF} 3 \_\mathrm{P} 7$ & ratio_$\gamma \_\mathrm{m}-\beta \_\mathrm{Fp} 1$ & cfC_C3_24_Hz & $\theta \_F z$ \\
\hline 8 & $\beta \_\mathrm{m}-\beta_{-} \mathrm{CP} 6 \_\mathrm{CP} 2$ & $\gamma \_\mathrm{m}-\theta \_\mathrm{AF} 3 \_\mathrm{P} 7$ & ratio_$\beta \_\mathrm{m}-\alpha_{-} \mathrm{Fp} 1$ & esc_F3 & $\alpha \_\mathrm{PO} 3$ \\
\hline 9 & $\alpha \_\mathrm{m}-\theta+01 \_\mathrm{CP} 2$ & $\gamma \_\mathrm{m}-\alpha \_$F3_Oz & $\gamma_{-} \mathrm{m}-\beta_{-} \mathrm{PO} 4$ & cfc_O2_14_Hz & Al_a_P7_P8 \\
\hline 10 & $\beta \_\mathrm{m}-\beta \_\mathrm{F} 4 \_\mathrm{CP} 2$ & $\theta \_\mathrm{m}-\theta \_\mathrm{Pz} \_\mathrm{PO} 4$ & ratio_$\beta \beta \_\mathrm{m}-\theta \_\mathrm{P} 8$ & cfc_FC1_42_Hz & $\theta_{-} \mathrm{O} 1$ \\
\hline 11 & $\beta \_\mathrm{m}-\theta \_\mathrm{AF} 3 \_\mathrm{Oz}$ & $\alpha \_\mathrm{m}-\alpha \_F p 1 \_\mathrm{Pz}$ & $\beta \_\mathrm{m}-\beta \_\mathrm{P} 3$ & cfc_FC1_43_Hz & $\beta \_\mathrm{PO} 3$ \\
\hline 15 & $\alpha \_\mathrm{m}-\theta$-01_O2 & $\beta \_\mathrm{m}-\alpha \_\mathrm{AF} 3 \_\mathrm{F} 4$ & $\beta \_\mathrm{m}-\alpha_{-} \mathrm{P} 3$ & cfc_CP1_30_Hz & $\mathrm{Al} \_\beta \_\mathrm{PO} 3 \_\mathrm{PO} 4$ \\
\hline 16 & $\alpha \_\mathrm{m}-\theta \_01 \_\mathrm{Cz}$ & $\gamma \_\mathrm{m}-\theta_{-} \mathrm{O} z_{-} \mathrm{O} 2$ & $\beta \_\mathrm{m}-\alpha_{-} \mathrm{PO} 4$ & cfc_CP1_24_Hz & Al_$\beta$ _FC5_FC6 \\
\hline 17 & $\alpha_{-} \mathrm{m}-\alpha_{-} \mathrm{CP} 1 \_\mathrm{PO} 4$ & $\beta \_\mathrm{m}-\alpha \_F 4 \_\mathrm{P} 8$ & $\beta \_\mathrm{m}-\beta \_\mathrm{T} 7$ & esc_F4 & Al_a_01_O2 \\
\hline 18 & $\gamma \_\mathrm{m}-\theta \_\mathrm{F} 3 \_\mathrm{O} 1$ & $\gamma \_\mathrm{m}-\alpha_{-} \mathrm{CP} 1 \_\mathrm{P} 7$ & $\beta \_\mathrm{m}-\theta \_\mathrm{T} 7$ & cfc_FC1_45_Hz & $\mathrm{Al} \_\beta \_\mathrm{F} 7 \_\mathrm{F} 8$ \\
\hline 19 & $\gamma \_\mathrm{m}-\beta \_\mathrm{P} 8 \_\mathrm{O} 2$ & $\gamma \_\mathrm{m}-\beta \_\mathrm{CP} 1 \_\mathrm{P} 7$ & ratio_$\_\beta \_\mathrm{m}-\alpha \_\mathrm{PO} 3$ & cfc_CP1_26_Hz & Al_t$\theta \_A F 3 \_A F 4$ \\
\hline 20 & $\alpha \_\mathrm{m}-\theta \_01 \_\mathrm{Fz}$ & $\beta \_\mathrm{m}-\beta \_\mathrm{CP} 5 \_\mathrm{T} 8$ & $\gamma \_\mathrm{m}-\theta_{-} \mathrm{PO} 4$ & cfc_CP1_29_Hz & $\alpha_{-} F z$ \\
\hline 21 & $\alpha \_\mathrm{m}-\theta \_\mathrm{F} 7 \_\mathrm{AF} 4$ & $\beta \_\mathrm{m}-\beta \_\mathrm{F} 7 \_\mathrm{P} 8$ & ratio_$\_\beta \_\mathrm{m}-\beta \_\mathrm{PO} 3$ & cfc_CP1_27_Hz & Al_$\beta \_P 3 \_P 4$ \\
\hline 22 & $\alpha \_\mathrm{m}-\alpha \_01 \_\mathrm{Cz}$ & $\gamma \_\mathrm{m}-\beta \_\mathrm{AF} 3 \_\mathrm{P} 7$ & ratio $\_\theta \_\mathrm{m}-\theta \_\mathrm{CP} 2$ & cfC_FC1_44_Hz & $\beta \_\mathrm{P} 3$ \\
\hline 23 & $\alpha \_\mathrm{m}-\theta \_01 \_\mathrm{Oz}$ & $\theta \_\mathrm{m}-\theta \_\mathrm{O} 1 \_\mathrm{Cz}$ & ratio_$\gamma_{-}$m- $\alpha_{-} F p 1$ & esc_AF3 & $\theta \_\mathrm{AF} 3$ \\
\hline
\end{tabular}

Feature names listed should be self explanatory. The "ratio" features correspond to the log-ratio ones between the video and baseline periods; "Al" corresponds to the asymmetry index between the indicated channels.

modulation coupling for affective state recognition. In fact, for the liking dimension $100 \%$ of the AMC features came from these two bands and this feature set resulted in the greatest improvement over the benchmark set (i.e., 1.9\% increase). Moreover, $\beta$ and $\alpha$ interactions were shown useful for dominance prediction in Liu and Sourina (2012). Here, 63\% of the AMI features corresponded to those bands with several $\beta \_\mathrm{m}-\alpha$ features appearing at the top. Interestingly, for the AMC features, all top 19 features corresponded to $\beta$ band interactions, with several coming from parietal regions, thus corroborating findings in Liu and Sourina (2012).

From the Tables, it can also be seen that the proposed normalization scheme over the baseline period was shown to be extremely important for the AME features, which unlike AMI and AMC, are energy-based features and not connectivity ones. For arousal, roughly $57 \%$ of the features corresponded to normalized features. For valence and liking they roughly corresponded to half of the top feature set. Normalization is important in order to remove participantspecific variability. Interestingly, only for the dominance dimension were normalized features seldom selected (20\%) and it was for this emotional primitive that the AME features showed to be most useful. When analyzing the high/low threshold used per subject, it was observed that for the dominance dimension, the standard deviation of the optimal threshold across participants was lower at 0.65 . For comparison purposes, the standard deviation for arousal (shown in Figure 3) was of 0.71. As such, since there was lower inter-subject variability for the dominance dimension, normalization was not as important. Overall, for the entire AMF set, channels that involved the frontal region provided several relevant features, thus confirming the importance of the frontal region for affective state recognition (Mikutta et al., 2012).

\subsection{Classification and Feature Fusion}

As shown in Table 5, all tested features and feature combinations resulted in balanced accuracy results significantly greater than chance. When all classifiers relied on the same input dimensionality and default parameters, the superiority of the proposed amplitude modulation features could be seen, particularly for the arousal, dominance and liking dimensions. In the case of equal dimensionality, fusion of AMF features did not result in any improvements over the individual amplitude modulation features, both for feature- and decision-level fusion. Notwithstanding, some improvement was seen when more features were explored. PAC features, in turn, were shown to be particularly useful for valence estimation. When PAC features were fused with benchmark and proposed AMF features, (i) feature-level fusion was shown to be particularly useful for arousal estimation, achieving results significantly better than 
TABLE 3 | Selected top-19 features for the dominance dimension.

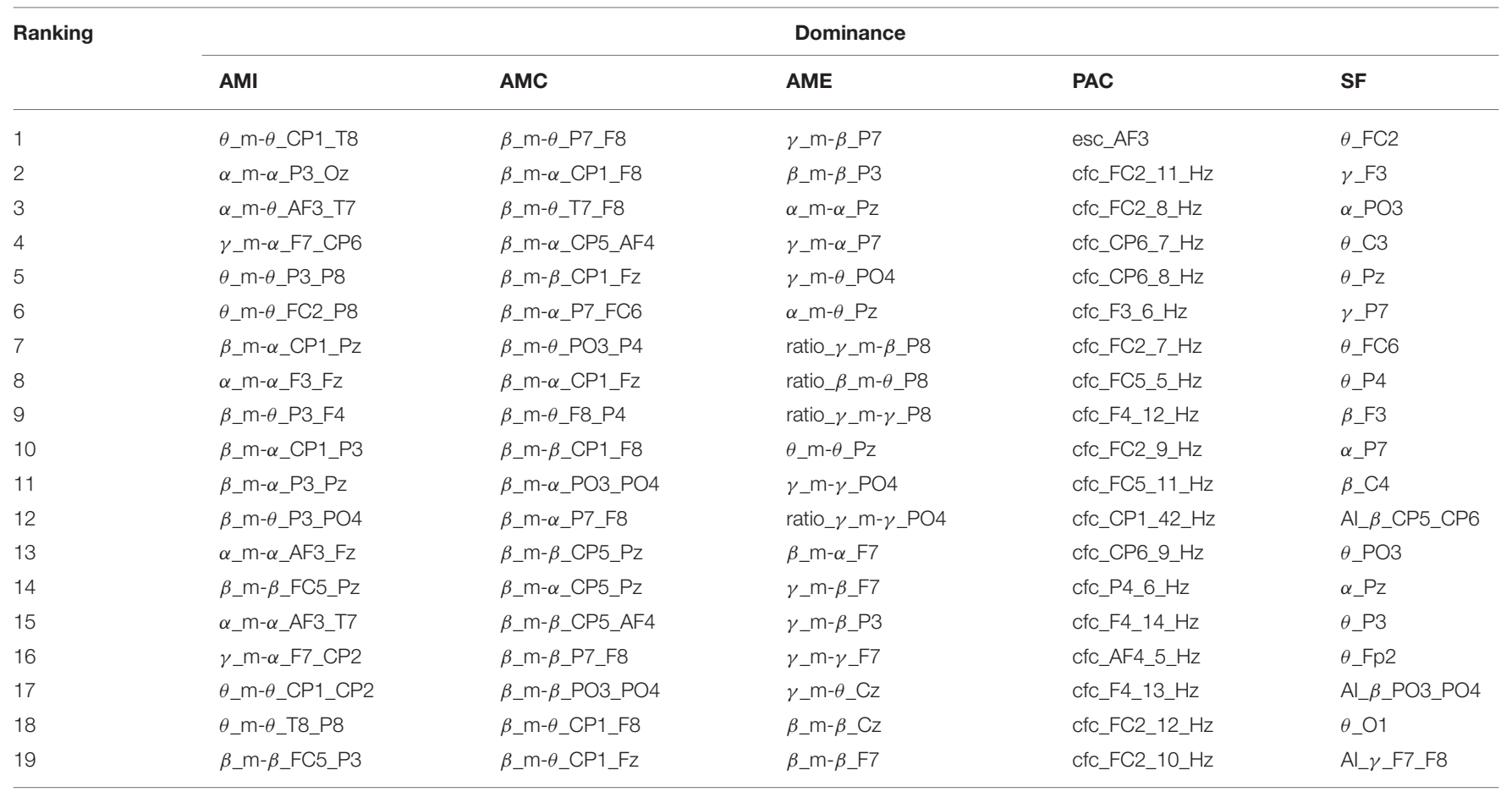

Feature names listed should be self explanatory. The "ratio" features correspond to the log-ratio ones between the video and baseline periods; "Al" corresponds to the asymmetry index between the indicated channels.

TABLE 4 | Selected top-21 features for the liking dimension.

\begin{tabular}{|c|c|c|c|c|c|}
\hline Ranking & \multicolumn{5}{|c|}{ Liking } \\
\hline 2 & $\beta \_\mathrm{m}-\alpha \_\mathrm{PO} 3 \_\mathrm{P} 8$ & $\beta \_\mathrm{m}-\beta \_01 \_T 8$ & $\gamma \_\mathrm{m}-\beta \_\mathrm{P} 7$ & cfC_FC1_7_Hz & $\theta \_$C3 \\
\hline 3 & $\alpha \_\mathrm{m}-\alpha \_01 \_\mathrm{Oz}$ & $\beta \_\mathrm{m}-\beta \_\mathrm{Pz} \_\mathrm{FC} 2$ & ratio_$\gamma_{-} m-\gamma \_$P8 & cfc_P7_29_Hz & $\beta_{-} \mathrm{P} 3$ \\
\hline 4 & $\gamma_{-} \mathrm{m}-\gamma_{-} \mathrm{Fp} 1 \_T 7$ & $\gamma \_\mathrm{m}-\beta \_\mathrm{Pz} \_\mathrm{AF} 4$ & $\gamma \_\mathrm{m}-\theta \_\mathrm{P} 3$ & cfc_PO4_42_Hz & $\beta \_$Т8 \\
\hline 7 & $\theta \_\mathrm{m}-\theta$-C3_P8 & $\beta \_\mathrm{m}-\theta$ $\theta$ FC1_O1 & ratio_$\gamma \_m-\theta$ _F3 & cfC_P7_31_Hz & $\alpha \_$F8 \\
\hline 8 & $\theta \_\mathrm{m}-\theta \_\mathrm{CP} 5 \_\mathrm{AF} 4$ & $\gamma \_\mathrm{m}-\theta \_\mathrm{Pz} \_\mathrm{AF} 4$ & $\alpha \_\mathrm{m}-\alpha_{-} \mathrm{CP} 1$ & cfc_PO4_39_Hz & $\beta \_\mathrm{PO} 3$ \\
\hline 9 & $\beta \_\mathrm{m}-\theta \_\mathrm{P} 3 \_\mathrm{AF} 4$ & $\gamma \_\mathrm{m}-\gamma_{-} \mathrm{AF} 3 \_\mathrm{Oz}$ & ratio_,$\beta \_\mathrm{m}-\theta \_\mathrm{P} 8$ & cfc_PO4_45_Hz & Al_ $\beta$ FC5_FC6 \\
\hline 10 & $\beta \_\mathrm{m}-\theta \_\mathrm{F} 7 \_\mathrm{AF} 4$ & $\gamma \_\mathrm{m}-\beta \_\mathrm{CP} 1 \_\mathrm{AF} 4$ & $\gamma \_\mathrm{m}-\theta \_\mathrm{F} 3$ & esc_F3 & $\beta \_\mathrm{AF3}$ \\
\hline 11 & $\theta \_\mathrm{m}-\theta \_\mathrm{P} 7 \_\mathrm{AF} 4$ & $\beta \_\mathrm{m}-\alpha \_01 \_\mathrm{T} 8$ & ratio $\_\beta \_\mathrm{m}-\beta \_\mathrm{C} 3$ & cfc_PO4_44_Hz & $\theta \_\mathrm{CP} 1$ \\
\hline 15 & $\theta \_\mathrm{m}-\theta \_\mathrm{CP} 1 \_\mathrm{AF} 4$ & $\gamma \_\mathrm{m}-\beta \_\mathrm{AF} 3 \_\mathrm{Oz}$ & ratio_$\gamma_{-} m-\gamma_{-}$FC6 & cfc_PO4_41_Hz & $\mathrm{Al} \_\beta \_F 7 \_F 8$ \\
\hline 16 & $\alpha \_\mathrm{m}-\theta \_\mathrm{O} z \_\mathrm{FC} 2$ & $\beta \_\mathrm{m}-\theta \_\mathrm{FC} 5 \_\mathrm{PO} 3$ & ratio_$\beta \_\mathrm{m}-\alpha \_F p 2$ & cfc_PO4_43_Hz & Al_$\beta \_P O 3 \_P O 4$ \\
\hline 17 & $\alpha \_\mathrm{m}-\theta \_\mathrm{P} 7 \_\mathrm{P} 8$ & $\gamma \_\mathrm{m}-\gamma_{-} \mathrm{CP} 1 \_\mathrm{AF} 4$ & ratio $\_\gamma \_\mathrm{m}-\beta \_\mathrm{P} 8$ & cfC_P7_27_Hz & $\theta_{-} \mathrm{FC6}$ \\
\hline 18 & $\beta \_\mathrm{m}-\beta \_\mathrm{PO} 3 \_\mathrm{P} 4$ & $\gamma \_\mathrm{m}-\alpha \_\mathrm{AF} 3 \_\mathrm{Oz}$ & $\beta \_\mathrm{m}-\theta \_\mathrm{P} 7$ & esc_P8 & $\beta_{-} \mathrm{FC5}$ \\
\hline 19 & $\theta \_\mathrm{m}-\theta \_\mathrm{Pz} \_\mathrm{CP} 6$ & $\beta \_\mathrm{m}-\theta \_\mathrm{F} 3 \_\mathrm{P} 8$ & ratio_$\theta-m-\theta \_P 7$ & cfC_FC1_8_Hz & Al_ $\theta \_F 7 \_F 8$ \\
\hline 20 & $\theta \_\mathrm{m}-\theta \_\mathrm{Pz} \_\mathrm{AF} 4$ & $\beta \_\mathrm{m}-\beta \_$F3_P8 & ratio_,$\beta \_\mathrm{m}-\beta \_\mathrm{F} 3$ & cfc_FC6_10_Hz & $\theta_{-} \mathrm{F} 4$ \\
\hline 21 & $\beta \_\mathrm{m}-\theta \_\mathrm{AF} 4$ _T8 & $\gamma \_\mathrm{m}-\alpha \_\mathrm{CP} 1 \_\mathrm{AF} 4$ & $\beta \_\mathrm{m}-\beta \_\mathrm{T} 7$ & cfC_P7_28_Hz & $\theta \_F z$ \\
\hline
\end{tabular}

Feature names listed should be self explanatory. The "ratio" features correspond to the log-ratio ones between the video and baseline periods; "Al" corresponds to the asymmetry index between the indicated channels. 
TABLE 5 | Performance comparison of SVM classifiers for different feature sets and fusion strategies.

\begin{tabular}{|c|c|c|c|c|c|c|c|c|c|}
\hline Feature class & Fusion type & Valence & $\%$ & Arousal & $\%$ & Dominance & $\%$ & Liking & $\%$ \\
\hline AMI & - & 0.604 & 4.4 & 0.583 & 5.6 & 0.564 & 4.1 & 0.626 & 1.1 \\
\hline AMC & - & 0.594 & 2.7 & 0.563 & 1.9 & 0.569 & 5.0 & 0.630 & 1.9 \\
\hline AME & - & 0.600 & 3.6 & 0.563 & 1.9 & 0.573 & 5.6 & 0.627 & 1.3 \\
\hline AMF & Feature-level & 0.594 & 2.7 & 0.583 & 5.6 & 0.566 & 4.4 & 0.624 & 0.9 \\
\hline PAC & - & 0.634 & 9.7 & 0.568 & 3.0 & 0.559 & 3.2 & 0.629 & 1.7 \\
\hline SF & - & 0.578 & - & 0.552 & - & 0.542 & - & 0.619 & - \\
\hline $\mathrm{AMF}+\mathrm{SF}+\mathrm{PAC}$ & Feature-level & 0.594 & 2.7 & 0.598 & 8.4 & 0.567 & 4.6 & 0.624 & 0.9 \\
\hline $\mathrm{AMI}+\mathrm{AMC}+\mathrm{AME}$ & Decision-level & 0.594 & 2.8 & 0.563 & 1.9 & 0.567 & 4.6 & 0.625 & 1.0 \\
\hline $\mathrm{AMF}+\mathrm{PAC}+\mathrm{SF}$ & Decision-level & 0.594 & 2.7 & 0.563 & 1.9 & 0.563 & 3.7 & 0.633 & 2.2 \\
\hline
\end{tabular}

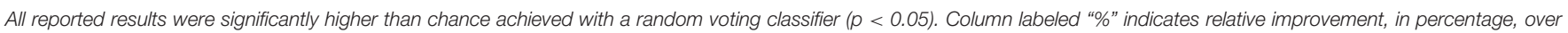
the SF baseline set.

the benchmark ( $p \leq 0.05$ ), and (ii) decision-level fusion was shown to be useful for liking prediction. Once varying input dimensionality was explored, the advantages of the proposed features over the benchmark became more evident, with gains as high as 8 and $20 \%$ being observed for the valence and dominance dimensions, respectively. Such results were significantly better than the benchmark ( $p \leq 0.05)$.

\subsection{Study Limitations}

This study has relied on the publicly available pre-processed DEAP database, which utilized a common average reference. Such referencing scheme could have introduced an artificial correspondence between nearby channels, thus potentially biasing the amplitude modulation and connectivity measures (Dezhong, 2001; Dezhong et al., 2005). By utilizing the multistage feature selection strategy, such biases were reduced, as feature redundancy was minimized and relevance was maximized. Moreover, from the relevant connections reported in the Tables, it can be seen that the majority of relevant connections are from electrodes that are sufficiently far apart, thus overcoming potential smearing contamination issues due to referencing. Moreover, as with many other machine learning problems, differences in data partitioning may lead to different top-selected features and, consequently, to varying performance results. This is particularly true for smaller datasets such as the one used herein. To test the sensitivity of data partitioning on feature selection, we randomly partitioned the $25 \%$ subset twice and explored the top selected features in each partition. For the AME features, for example, and the valence dimension, it was found that 13 of the top 23 features coincided for the two partitions. While this number is not very high, it is encouraging and future work should explore the use of boosting strategies and/or alternate data partitioning schemes to improve this.

\section{CONCLUSIONS}

In this work, experimental results with the publicly available DEAP database showed the EEG amplitude modulation based feature sets such as amplitude-amplitude crossfrequency modulation coupling features, as well as linear and nonlinear connection between multiple electrode pairs outperformed benchmark measures based on spectral power by as much as maximum $20 \%$ for dominance. Moreover, phaseamplitude coupling of EEG and GSR signals outperformed the benchmark by over $9 \%$ and when fused with the proposed amplitude modulation features, further gains in arousal and liking prediction were observed. Such findings suggest the importance of the proposed features for affective state recognition and signal the importance of EEG amplitude modulation for affective tagging of music video clips and content.

\section{ETHICS STATEMENT}

This study relied on publicly available data collected by others. Details about the database can be found at: Koelstra et al. (2012).

\section{AUTHOR CONTRIBUTIONS}

AC performed data analysis and prepared the manuscript. RG assisted with data analysis and classification. SJ assisted with connectivity analysis and manuscript writing. TF contributed the study design, project supervision, and manuscript preparation. All authors read and approved the final manuscript.

\section{FUNDING}

The authors would like to thank NSERC for funding this project via its Discovery and PERSWADE CREATE Programs.

\section{ACKNOWLEDGMENTS}

The authors would like to thank Adriano Tort of the Federal University of Rio Grande do Norte (Brazil) for sharing the ModI Matlab code, as well as NSERC for the funding. 


\section{REFERENCES}

Aftanas, L., and Golocheikine, S. (2001). Human anterior and frontal midline theta and lower alpha reflect emotionally positive state and internalized attention: high-resolution eeg investigation of meditation. Neurosci. Lett. 310, 57-60. doi: 10.1016/S0304-3940(01)02094-8

Arndt, S., Antons, J., Gupta, R., Schleicher, R., Moller, S., and Falket, T. H. (2013). "The effects of text-to-speech system quality on emotional states and frontal alpha band power," in IEEE International Conference on Neural Engineering (San Diego, CA), 489-492.

Arnulfo, G., Hirvonen, J., Nobili, L., Palva, S., and Palva, J. M. (2015). Phase and amplitude correlations in resting-state activity in human stereotactical EEG recordings. NeuroImage 112, 114-127. doi: 10.1016/j.neuroimage.2015.02.031

Balconi, M., and Lucchiari, C. (2008). Consciousness and arousal effects on emotional face processing as revealed by brain oscillations. A gamma band analysis. Int. J. Psychophysiol. 67, 41-46. doi: 10.1016/j.ijpsycho.2007.10.002

Bersak, D., McDarby, G., Augenblick, N., McDarby, P., McDonnell, D., McDonald, B., et al. (2001). "Intelligent biofeedback using an immersive competitive environment," in Paper at the Designing Ubiquitous Computing Games Workshop at UbiComp (Atlanta, GA).

Boashash, B. (1991). Time-Frequency Signal Analysis. Prentice Hall.

Bos, D. P.-O., Reuderink, B., van de Laar, B., Gurkok, H., Muhl, C., Poel, M., et al. (2010). "Human-computer interaction for BCI games: usability and user experience," in Proceedings of the IEEE International Conference on Cyberworlds (Singapore), 277-281. doi: 10.1109/CW.2010.22

Bradley, M., and Lang, P. (1994). Measuring emotion: the self-assessment manikin and the semantic differential. Behav. Ther. Exp. Psychiatry 25, 49-59. doi: 10.1016/0005-7916(94)90063-9

Canolty, R. T., Cadieu, C. F., Koepsell, K., Knight, R. T., and Carmena, J. M. (2012). Multivariate phase-amplitude cross-frequency coupling in neurophysiological signals. IEEE Trans. Biomed. Eng. 59, 8-11. doi: 10.1109/TBME.2011.2172439

Canolty, R. T., Edwards, E., Dalal, S. S., Soltani, M., Nagarajan, S. S., Kirsch, H. E., et al. (2006). High gamma power is phase-locked to theta oscillations in human neocortex. Science 313, 1626-1628. doi: 10.1126/science.1128115

Chik, D. (2013). Theta-alpha cross-frequency synchronization facilitates working memory control-a modeling study. SpringerPlus 2:14. doi: 10.1186/21931801-2-14

Clerico, A., Gupta, R., and Falk, T. (2015). "Mutual information between interhemispheric EEG spectro-temporal patterns: a new feature for automated affect recognition," in IEEE Neural Engineering Conference (Montpellier), 914-917.

Coan, J., and Allen, J. (2004). Frontal EEG asymmetry as a moderator and mediator of emotion. Biol. Psychol. 67, 7-50. doi: 10.1016/j.biopsycho.2004. 03.002

Cohen, I., Sebe, N., Garg, A., Chen, L. S., and Huang, T. S. (2003). Facial expression recognition from video sequences: temporal and static modeling. Comput. Vis. Image Understand. 91, 160-187. doi: 10.1016/S1077-3142(03)00081-X

Cohen, M. X. (2008). Assessing transient cross-frequency coupling in eeg data. J. Neurosci. Methods 168, 494-499. doi: 10.1016/j.jneumeth.2007.10.012

Daly, I., Malik, A., Hwang, F., Roesch, E., Weaver, J., Kirke, A., et al. (2014). Neural correlates of emotional responses to music: an EEG study. Neurosci. Lett. 573, 52-57. doi: 10.1016/j.neulet.2014.05.003

Dastgheib, Z. A., Pouya, O. R., Lithgow, B., and Moussavi, Z. (2016). "Comparison of a new ad-hoc classification method with support vector machine and ensemble classifiers for the diagnosis of meniere's disease using evestg signals," in IEEE Canadian Conference on Electrical and Computer Engineering (Vancouver, BC).

Davidson, R., Schwartz, G., Saron, C., Bennett, J., and Goleman, D. J. (1979). Frontal versus parietal EEG asymmetry during positive and negative affect. Psychophysiology 16, 202-203.

Davidson, R. J. (2004). What does the prefrontal cortex "do" in affect: perspectives on frontal EEG asymmetry research. Biol. Psychol. 67, 219-234. doi: 10.1016/j.biopsycho.2004.03.008

Davidson, R. J., and Tomarken, A. J. (1989). Laterality and emotion: an electrophysiological approach. Handb. Neuropsychol. 3, 419-441.

De Martino, B., Kumaran, D., Seymour, B., and Dolan, R. J. (2006). Frames, biases, and rational decision-making in the human brain. Science 313, 684-687. doi: $10.1126 /$ science. 1128356
Dezhong, Y. (2001). A method to standardize a reference of scalp EEG recordings to a point at infinity. Physiol. Meas. 22, 693-711. doi: 10.1088/09673334/22/4/305

Dezhong, Y., Wang, L., Oostenveld, R., Nielsen, K. D., Arendt-Nielsen, L., and Chen, A. C.(2005). A comparative study of different references for EEG spectral mapping: the issue of the neutral reference and the use of the infinity reference. Physiol. Meas. 26, 173-184. doi: 10.1088/0967-3334/26/3/003

Falk, T. H., Fraga, F. J., Trambaiolli, L., and Anghinah, R. (2012). EEG amplitude modulation analysis for semi-automated diagnosis of alzheimer's disease. EURASIP J. Adv. Signal Process. 2012:192. doi: 10.1186/1687-6180-2012-192

Gabor, D. (1946). Theory of communication. part 1: the analysis of information. J. IEE III 93, 429-441. doi: 10.1049/ji-3-2.1946.0074

Gupta, R., and Falk, T. (2015). "Affective state characterization based on electroencephalography graph-theoretic features," in IEEE Neural Engineering Conference (Montpellier), 577-580.

Gupta, R., Laghari, U. R., and Falk, T. H., (2016). Relevance vector classifier decision fusion and EEG graph-theoretic features for automatic affective state characterization. Neurocomputing 174, 875-884. doi: 10.1016/j.neucom. 2015.09.085

Hadjidimitriou, S. K., and Hadjileontiadis, L. J. (2012). Toward an EEG-based recognition of music liking using time-frequency analysis. IEEE Trans. Biomed. Eng. 59, 3498-3510. doi: 10.1109/TBME.2012.2217495

Hagemann, D., Naumann, E., Lürken, A., Becker, G., Maier, S., and Bartussek, D. (1999). EEG asymmetry, dispositional mood and personality. Pers. Indiv. Diff. 27, 541-568. doi: 10.1016/S0191-8869(98)00263-3

Hamm, J., Pinkham, A., Gur, R. C., Verma, R., and Kohler, C. G. (2014). Dimensional information-theoretic measurement of facial emotion expressions in schizophrenia. Schizophr. Res. Treat. 2014:243907. doi: 10.1155/2014/243907

Heger, D., Putze, F., and Schultz, T. (2010). "Online workload recognition from EEG data during cognitive tests and human-machine interaction," in Advances in Artificial Intelligence: 33rd Annual German Conference on AI, eds R. Dillmann, J. Beyerer, U. D. Hanebeck, and T. Schultz (Berlin; Heidelberg: Springer), 410-417.

Jenke, R., Peer, A., and Buss, M. (2014). Feature extraction and selection for emotion recognition from EEG. IEEE Trans. Affect. Comput. 5, 327-339. doi: 10.1109/TAFFC.2014.2339834

Kar, R., Konar, A., Chakraborty, A., and Nagar, A. K. (2014). "Detection of signaling pathways in human brain during arousal of specific emotion," in 2014 International Joint Conference on Neural Networks (IJCNN) (Beijing: IEEE), 3950-3957.

Kensinger, E. A. (2004). Remembering emotional experiences: the contribution of valence and arousal. Rev. Neurosci. 15, 241-252. doi: 10.1515/REVNEURO. 2004.15.4.241

Khushaba, R. N., Greenacre, L., Kodagoda, S., Louviere, J., Burke, S., and Dissanayake, G. (2012). Choice modeling and the brain: a study on the electroencephalogram (eeg) of preferences. Exp. Syst. Appl. 39, 12378-12388. doi: 10.1016/j.eswa.2012.04.084

Kierkels, J., Soleymani, M., and Pun, T. (2009). "Queries and tags in affect-based multimedia retrieval," in IEEE International Conference on Multimedia and Expo (New York, NY: IEEE), 1436-1439.

Koelstra, S., Mühl, C., Soleymani, M., and Patras, I. (2012). DEAP: a database for emotion analysis using physiological signals. IEEE Trans. Affect. Comput. 3, 18-31. doi: 10.1109/T-AFFC.2011.15

Koelstra, S., and Patras, I. (2013). Fusion of facial expressions and eeg for implicit affective tagging. Image Vis. Comput. 31, 164-174. doi: 10.1016/ j.imavis.2012.10.002

Kothe, C. A., and Makeig, S. (2011). "Estimation of task workload from EEG data: new and current tools and perspectives," in International Conference of the IEEE EMBS (Boston, MA: IEEE), 6547-6551.

Kroupi, E., Vesin, J.-M., and Ebrahimi, T. (2013). "Phase-amplitude coupling between EEG and EDA while experiencing multimedia content," in International Conference on Affective Computing and Intelligent Interaction (Geneva: IEEE), 865-870.

Kroupi, E., Vesin, J.-M., and Ebrahimi, T. (2014). Implicit affective profiling of subjects based on physiological data coupling. Brain Comput. Interf. 1, 85-98. doi: 10.1080/2326263X.2014.912882

Kullback, S., and Leibler, R. A. (1951). On information and sufficiency. Ann. Math. Stat. 22, 79-86. doi: 10.1214/aoms/1177729694 
Le Van Quyen, M., Foucher, J., Lachaux, J.-P., Rodriguez, E., Lutz, A., Martinerie, J., et al. (2001). Comparison of hilbert transform and wavelet methods for the analysis of neuronal synchrony. J. Neurosci. Methods 111, 83-98. doi: 10.1016/S0165-0270(01)00372-7

Lee, N., Broderick, A. J., and Chamberlain, L. (2007). What is "neuromarketing?" a discussion and agenda for future research. Int. J. Psychophysiol. 63, 199-204. doi: 10.1016/j.ijpsycho.2006.03.007

Leeb, R., Friedman, D., Slater, M., and Pfurtscheller, G. (2012). "A tetraplegic patient controls a wheelchair in virtual reality," in BRAINPLAY 07 BrainComputer Interfaces and Games Workshop at ACE (Advances in Computer Entertainment) 2007, 37.

Li, H., Li, Y., and Guan, C. (2006). An effective bci speller based on semisupervised learning. Conf. Proc. IEEE Eng. Med. Biol. Soc. 1, 1161-1164. doi: 10.1109/IEMBS.2006.260694

Lisetti, C. L., and Nasoz, F. (2004). Using noninvasive wearable computers to recognize human emotions from physiological signals. EURASIP J. Appl. Signal Process. 2004, 1672-1687. doi: 10.1155/S1110865704406192

Liu, Y., and Sourina, O. (2012). "EEG-based dominance level recognition for emotion-enabled interaction," in IEEE International Conference on Multimedia and Expo (Melbourne, VIC), 1039-1044.

Loewenstein, G., and Lerner, J. S. (2003). "The role of affect in decision making," in Handbook of Affective Science, eds R. Davidson, H. Goldsmith, and K. Scherer (Oxford: Oxford University Press), 619-642.

Mantini, D., Perrucci, M. G., Del Gratta, C., Romani, G. L., and Corbetta, M. (2007). Electrophysiological signatures of resting state networks in the human brain. Proc. Natl. Acad. Sci. U.S.A. 104, 13170-13175. doi: $10.1073 /$ pnas.0700668104

Marg, E. (1995). Descartes'error: emotion, reason, and the human brain. Optomet. Vis. Sci. 72, 847-848

Mikutta, C., Altorfer, A., Strik, W., and Koenig, T. (2012). Emotions, arousal, and frontal alpha rhythm asymmetry during beethoven's 5th symphony. Brain Topogr. 25, 423-430. doi: 10.1007/s10548-012-0227-0

Moore Jackson, M., and Mappus, R. (2010). “Applications for brain-computer interfaces," in Brain-Computer Interfaces and Human-Computer Interaction Series, eds D. Tan and A. Nijholt (London: Springer), 89-103.

Mühl, C., Allison, B., Nijholt, A., and Chanel, G. (2014). A survey of affective brain computer interfaces: principles, state-of-the-art, and challenges. Brain Comput. Interf. 1, 66-84. doi: 10.1080/2326263X.2014.912881

Müller, M. M., Keil, A., Gruber, T., and Elbert, T. (1999). Processing of affective pictures modulates right-hemispheric gamma band EEG activity. Clin. Neurophysiol. 110, 1913-1920. doi: 10.1016/S1388-2457(99)00151-0

Nasoz, F., Lisetti, C. L., Alvarez, K., and Finkelstein, N. (2003). "Emotion recognition from physiological signals for user modeling of affect," in Proceedings of the 3rd Workshop on Affective and Attitude User Modelling (Pittsburgh, PA).

Onslow, A. C., Bogacz, R., and Jones, M. W. (2011). Quantifying phase-amplitude coupling in neuronal network oscillations. Prog. Biophys. Mol. Biol. 105, 49-57. doi: 10.1016/j.pbiomolbio.2010.09.007

Pedregosa, F., Varoquaux, G., Gramfort, A., Michel, V., Thirion, B., Grisel, O., et al. (2011). Scikit-learn: Machine Learning in Python. J. Mach. Learn. Res. $12,2825-2830$

Peng, H., Long, F., and Ding, C. (2005). Feature selection based on mutual information criteria of max-dependency, max-relevance, and min-redundancy. IEEE Trans. Patt. Anal. Mach. Intell. 27, 1226-1238. doi: 10.1109/TPAMI.2005.159
Picard, R. W. (2000). Affective Computing. Cambridge: MIT Press.

Picard, R. W., and Healey, J. (1997). Affective wearables. Pers. Technol. 1, 231-240. doi: $10.1007 / \mathrm{BF} 01682026$

Preece, J., Rogers, Y., Sharp, H., Benyon, D., Holland, S., and Carey, T. (1994) Human-Computer Interaction. Addison-Wesley Longman Ltd.

Russell, J. A. (1980). A circumplex model of affect. J. Pers. Soc. Psychol. 39:1161. doi: $10.1037 /$ h0077714

Samiee, S., Donoghue, T., Tadel, F., and Baillet, S. Phase-Amplitude Coupling. Available online at: http://neuroimage.usc.edu/brainstorm/Tutorials/TutPac

Schölkopf, B., and Smola, A. J. (2002). Learning with Kernels: Support Vector Machines, Regularization, Optimization, and Beyond. Cambridge: MIT Press.

Schutter, D. J. L. G., and Knyazev, G. G. (2012). Cross-frequency coupling of brain oscillations in studying motivation and emotion. Motiv. Emot. 36, 46-54. doi: 10.1007/s11031-011-9237-6

Schutter, D. J. L. G., Putman, P., Hermans, E., and van Honk, J. (2001). Parietal electroencephalogram beta asymmetry and selective attention to angry facial expressions in healthy human subjects. Neurosci. Lett. 314, 13-16. doi: 10.1016/S0304-3940(01)02246-7

Shan, M.-K., Kuo, F.-F., Chiang, M.-F., and Lee, S.-Y. (2009). Emotion-based music recommendation by affinity discovery from film music. Exp. Syst. Appl. 36, 7666-7674. doi: 10.1016/j.eswa.2008.09.042

Smith, Z. M., Delgutte, B., and Oxenham, A. J. (2002). Chimaeric sounds reveal dichotomies in auditory perception. Nature 416, 87-90. doi: 10.1038/ $416087 \mathrm{a}$

Sorensen, H., and Kjaer, T. (2013). A brain-computer interface to support functional recovery. Clin. Recov. CNS Damage 32, 95-100. doi: 10.1159/ 000346430

Sörnmo, L., and Laguna, P. (2005). Bioelectrical Signal Processing in Cardiac and Neurological Applications. San Diego, CA: Academic Press.

Tort, A. B., Komorowski, R., Eichenbaum, H., and Kopell, N. (2010). Measuring phase-amplitude coupling between neuronal oscillations of different frequencies. J. Neurophysiol. 104, 1195-1210. doi: 10.1152/jn.001 06.2010

Wang, X.-W., Nie, D., and Lu, B.-L. (2011). "EEG-based emotion recognition using frequency domain features and support vector machines," in Neural Information Processing (Berlin: Springer), 734-743.

Wu, D., and Parsons, T. D. (2011). "Active class selection for arousal classification," in Affective Computing and Intelligent Interaction (Memphis, TN: Springer), $132-141$.

Xielifuguli, K., Fujisawa, A., Kusumoto, Y., Matsumoto, K., and Kita, K. (2014). Pleasant/unpleasant filtering for affective image retrieval based on crosscorrelation of EEG features. Appl. Comput. Intell. Soft Comput. 2014:415187. doi: $10.1155 / 2014 / 415187$

Conflict of Interest Statement: The authors declare that the research was conducted in the absence of any commercial or financial relationships that could be construed as a potential conflict of interest.

Copyright (c) 2018 Clerico, Tiwari, Gupta, Jayaraman and Falk. This is an openaccess article distributed under the terms of the Creative Commons Attribution License (CC BY). The use, distribution or reproduction in other forums is permitted, provided the original author(s) or licensor are credited and that the original publication in this journal is cited, in accordance with accepted academic practice. No use, distribution or reproduction is permitted which does not comply with these terms. 